This document is confidential and is proprietary to the American Chemical Society and its authors. Do not copy or disclose without written permission. If you have received this item in error, notify the sender and delete all copies.

\title{
Efficient Coupling of Nanoparticles to Electrochemically Exfoliated Graphene
}

\begin{tabular}{|r|l|}
\hline Journal: & Journal of the American Chemical Society \\
\hline Manuscript ID: & ja-2015-02284t.R1 \\
\hline Manuscript Type: & Article \\
\hline Date Submitted by the Author: & 06-Apr-2015 \\
\hline Complete List of Authors: & $\begin{array}{l}\text { Wei, Wei; Max-Planck-Institut für Polymerforschung, } \\
\text { Wang, Gang; Max Planck Institute for Polymer Research, } \\
\text { Yang, Sheng; Max Planck Institute for Polymer Research, } \\
\text { Feng, Xinliang; Technische Universität Dresden, Center for Advancing } \\
\text { Electronics Dresden \& Department of Chemistry and Food Chemistry } \\
\text { Müllen, Klaus; Max Planck Institute for Polymer Research, }\end{array}$ \\
\hline
\end{tabular}

SCHOLARONE

Manuscripts 


\title{
Efficient Coupling of Nanoparticles to Electrochemically
} Exfoliated Graphene

\author{
Wei Wei, ${ }^{\dagger}$ Gang Wang, ${ }^{\dagger}$ Sheng Yang, ${ }^{\dagger}$ Xinliang Feng, ${ }^{*,+}$ and Klaus Müllen*,† \\ ${ }^{\dagger}$ Max Planck Institute for Polymer Research, Ackermannweg 10, Mainz 55128, Germany \\ ${ }^{\ddagger}$ Center for Advancing Electronics Dresden (cfaed) \& Department of Chemistry and Food Chemistry, \\ Technische Universität Dresden, Dresden 01062, Germany
}

\begin{abstract}
Electrochemically exfoliated graphene (EEG) is a new generation of high-quality graphene that holds great promise for the construction of hybrid materials. However, the assembly of EEG hybrids with well-defined nanostructures has remained a major challenge. In this study, we demonstrate a bottom-up approach towards the assembly of EEG sheets with a series of functional nanoparticles $\left(\mathrm{Si}, \mathrm{Fe}_{3} \mathrm{O}_{4}\right.$ and $\mathrm{Pt} \mathrm{NPs}$ ) into two-dimensional (2D) sandwich-like hybrid nanostructures. Polyaniline in the emeraldine base form (PANB) functions as a versatile dopant to couple NPs onto EEG through either electrostatic interactions or hydrogen bonding. This protocol enables processing and assembly of EEG using an economical pathway, for which we further demonstrate the potential application of EEG-Si hybrids as high-performance anode material for lithium storage.
\end{abstract}

\section{INTRODUCTION}

Graphene has been the focus of materials science and applications due to its fascinating properties such as high electrical conductivity, exceptional mechanical strength and chemical stability. ${ }^{1}$ Integration of graphene with various functional components is of great importance in materials synthesis because the formation of graphene hybrids (GHs), preferably by a controlled assembly procedure, can lead to materials with unconventional properties and diverse applications. ${ }^{2-4}$ To date, graphene oxide (GO) is the most popular building block for the construction of GHs with tailorable geometries, such as core-shells, ${ }^{5}$ two-dimensional (2D) sandwich-like structures, ${ }^{6}$ and three-dimensional (3D) foams. ${ }^{7}$ Because of the presence of oxygen containing functional groups, the engineering of a GO surface through either covalent (chemical modification and grafting) ${ }^{8}$ or non-covalent $(\pi-\pi$ stacking, hydrogen bonding, electrostatic interactions) $)^{9}$ approaches is feasible to anchor various organic or inorganic species. However, the production of GO involves an aggressive oxidation process that destroys the electrical conductivity of graphene due to the inevitable disruption of $\mathrm{sp}^{2}$-carbon 
conjugation. ${ }^{10}$ Although electrical conductivity can be partially recovered by thermal or chemical reduction, ${ }^{11}$ the quality of reduced GO remains substantially inferior to that of pristine graphene. Within the scope of the production techniques for graphene (e.g., mechanical cleavage, liquid-phase exfoliation, and chemical vapor deposition, amongst others), electrochemical exfoliation of graphite emerges as one of the most desirable routes to prepare high-quality graphene on a large scale under mild conditions. ${ }^{12-14}$ Recently, mass production of electrochemically exfoliated graphene (EEG) with low defect density and remarkable electronic properties in aqueous solutions was realized which opened enormous opportunities to fabricate various GHs for advanced applications. ${ }^{15,16}$

Assembly of EEG into GHs requires the individual dispersion of EEG in a solvent medium and strong interface interactions between EEG and the functional compounds. Unfortunately, because of its hydrophobic nature and inert surface properties, EEG can only be dispersed in polar aprotic solvents (e.g., N,N-dimethyl formamide (DMF) and $N$-methyl-2-pyrrolidone (NMP)) which is a major obstacle for solution processing and the assembly of EEG. ${ }^{16}$ Noncovalent functionalization of EEG offers an alternative solution. ${ }^{17}$ However, the presence of foreign stabilizers (e.g., surfactants or polymers) is undesirable for most applications due to their limited electronic conductivity and poor stability while the complete removal of residual stabilizers requires tedious purification or pyrolysis. ${ }^{18,19}$ Therefore, overcoming these limitations to simultaneously construct EEG assemblies or hybrids with well-defined nanostructures and to retain the superior properties of EEG has become the primary challenge for the development of hybrid materials based on EEG.

In this study, we present a bottom-up strategy to construct a series of novel 2D hybrids with EEG nanosheets sandwiched by various colloidal nanoparticles (CNPs). Polyaniline (PANI), in the emeraldine base form (PANB) is employed as a bi-functional linker to bridge the EEG and CNP counterparts. The aromatic backbone chains of PANB spontaneously adsorb onto the EEG surface via $\pi-\pi$ interactions, whereas the exposed amine/imine groups serve as reactive sites to couple with CNPs via electrostatic interactions and hydrogen bonding. Importantly, the conductivity of the PANB components can be recovered by a simple protonic acid doping method, accompanied by a conductivity enhancement of the resulting EEG hybrids. This strategy can be applied to fabricate various strongly coupled 2D graphene hybrids, including EEG-Si, EEG-Fe ${ }_{3} \mathrm{O}_{4}$ and EEG-Pt, which are interesting for a variety of possible applications. As exemplified by the use of EEG-Si anode material for lithium storage, a high initial reversible capacity of $2357 \mathrm{mAh} \mathrm{g}^{-1}$ at $105 \mathrm{~mA} \mathrm{~g}^{-1}$ and excellent cycling stability 
( $86 \%$ capacity retention after 100 cycles at $\left.1 \mathrm{~A} \mathrm{~g}^{-1}\right)$ are achieved. Even at a high current density of $8.4 \mathrm{~A} \mathrm{~g}^{-1}$, a reversible capacity of $460 \mathrm{mAh} \mathrm{g}^{-1}$ is maintained with excellent cycling stability.

\section{口 RESULTS AND DISCUSSION}

Assembly of EEG-CNP Hybrids. PANI is known to exist in several different oxidation states including reduced, half-oxidized, and completely oxidized polymers, ${ }^{20}$ as shown in Figure S1. PANI-derived materials were generally obtained as the emeraldine salt (PANS), ${ }^{21}$ with less focus on the utilization of the solution-processable polymer form, the emeraldine base (PANB). Compared with other oxidation states, PANB exhibits multiple advantages for EEG functionalization and processing. First, PANB is best suited for processing due to its high solubility in polar aprotic solvents (e.g., DMF and NMP ${ }^{22}$ which allows for efficient adsorption and non-covalent functionalization of EEG. Second, PANB contains exposed amine and imine groups which provide a sufficient number of binding sites to interact with foreign species. Third, the unique electrical switching property of PANB enhances the electrical conductivity of the EEG hybrids by simple protonic acid doping (e.g., by $\mathrm{HCl}){ }^{20}$
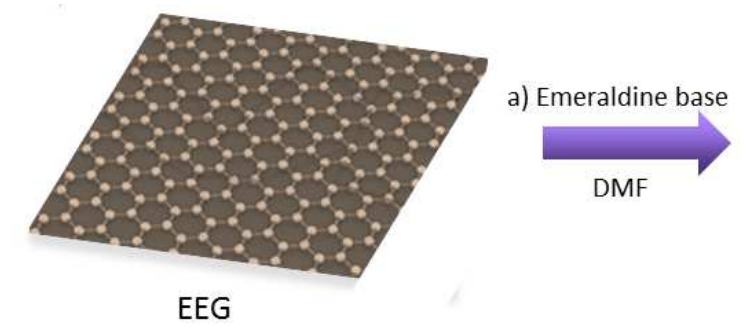

EEG

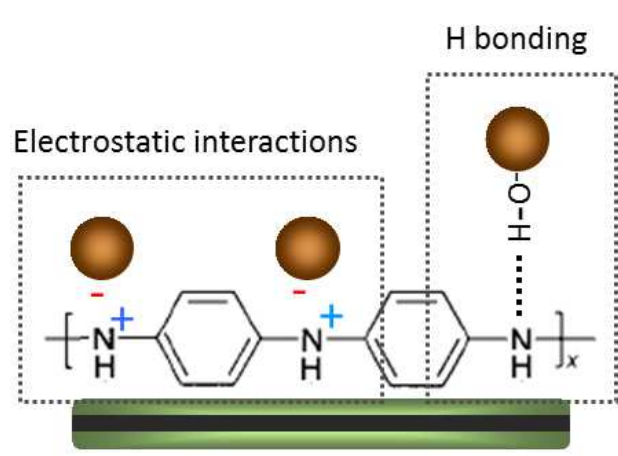

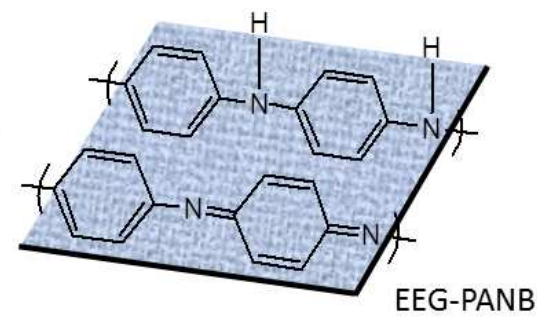

b) Colloidal NPS/ Assembly

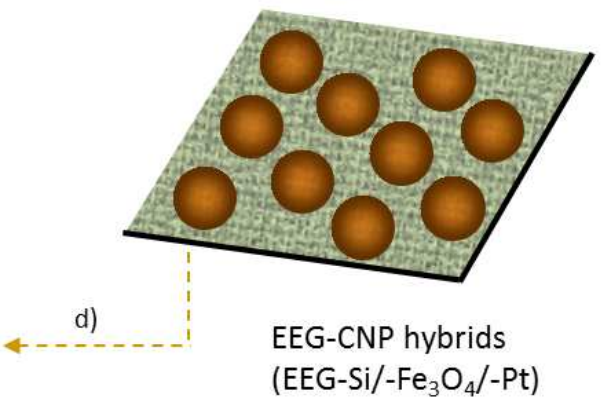

Figure 1. Synthetic procedure for EEG-CNP hybrids. a) EEG is functionalized with emeraldine base (PANB) in DMF solutions via $\pi-\pi$ interactions; b) EEG-PANB sheets are assembled with a variety of colloidal nanoparticles (CNPs, e.g., $\mathrm{Si}, \mathrm{Fe}_{3} \mathrm{O}_{4}, \mathrm{Pt}$ ) through electrostatic interactions and hydrogen bonding; c) Protonation of PANB with $\mathrm{HCl}$ gives rise to emeraldine salt (PANS) and promotes electrical conductivity of EEG hybrids; d) Simplified cross-section view of interactions between EEG and CNPs. 
The overall synthetic procedure for the 2D EEG-CNP hybrids is illustrated in Figure 1. EEG was first prepared according to a modified procedure via electrochemical exfoliation of graphite. ${ }^{16}$ Briefly, a constant voltage $(10 \mathrm{~V})$ was applied to a two-electrode system with a sodium methanesulfonate aqueous solution $(0.1 \mathrm{M})$ as the electrolyte and a graphite flake and platinum wire as the working and counter electrodes, respectively. The voltage was maintained constant for $10 \mathrm{~min}$ to complete the exfoliation process. The exfoliated product was collected, washed with water and dispersed in DMF by sonication. PANB-functionalized EEG (EEG-PANB) was prepared by mixing EEG and the PANB component in DMF with a concentration of 0.1 and $0.2 \mathrm{mg} \mathrm{mL}^{-1}$, respectively. In this step, the PANB molecules were expected to anchor onto the EEG surface through $\pi-\pi$ interactions. Subsequently, CNPs (e.g., $\mathrm{Si}, \mathrm{Fe}_{3} \mathrm{O}_{4}$ and $\mathrm{Pt}$ ) containing a variety of surface functionalities were added to the EEG-PANB dispersion in DMF. During this process, CNPs were bound to the amine/imine groups of PANB and assembled into EEG-PANB via electrostatic interactions and hydrogen bonding. Subsequent protonic acid doping with $\mathrm{HCl}(0.5 \mathrm{M})$ generated EEG-CNPs with sandwich-like nanostructures.
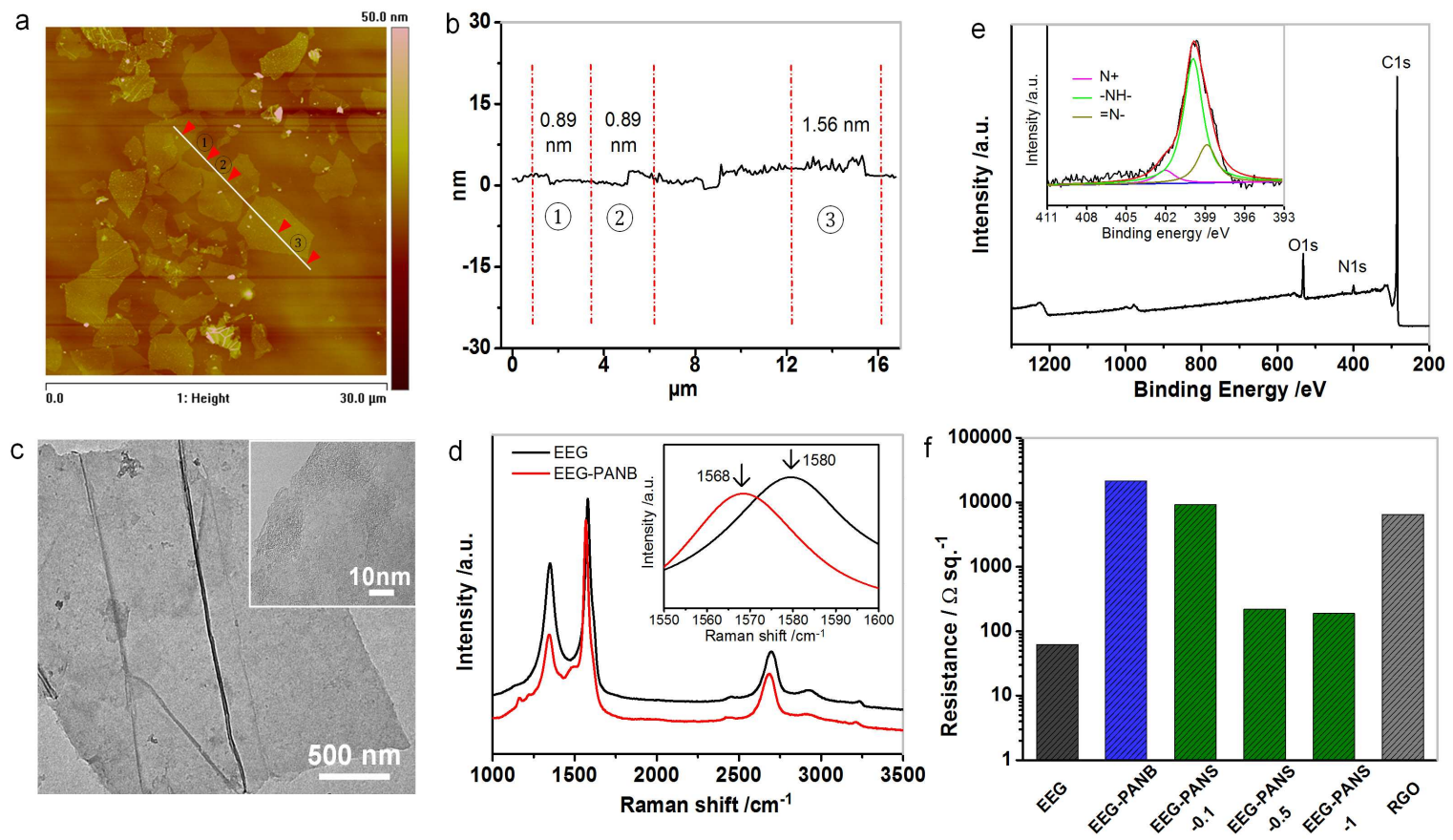

Figure 2. Characterizations of EEG and EEG-PANB. (a) AFM image of EEG sheets; (b) Height profile of EEG obtained around the white line in (a); (c) Typical TEM and HR-TEM (inset) images of EEG-PANB; (d) Raman spectra of EEG and EEG-PANB. Inset shows the shift of the G peak; (e) XPS spectra and N1s XPS spectra (inset) of EEG-PANB; (f) Resistance comparison of EEG, RGO, and EEG-PANB before and after doping with $\mathrm{HCl}$ at various concentrations. The film resistance decreases with an increase in $\mathrm{HCl}$ concentration, indicating the increased protonation degree and electrical conductivity of EEG-PANS. 
Morphological and Structural Characterization of EEG-PANB. The microstructure of the EEG sheets was examined by scanning electron microscopy (SEM) and transmission electron microscopy (TEM). As shown in Figure S2, freestanding sheets with lateral sizes ranging from hundreds of nanometers to several micrometers are observed. In certain cases, the sheet edges tend to scroll and fold slightly which is a behavior associated with the flexible and corrugated nature of graphene. The typical atomic force microscopy (AFM) image reveals that EEG has a flake structure (Figure 2a), and the height profile verifies that EEG is primarily composed of thin sheets ( $\leq 3$ layers) with a mean thickness of $0.89 \mathrm{~nm}$ and $1.56 \mathrm{~nm}$ for the monolayer and bilayer sheets, respectively (Figure 2b). Stirring of EEG and PANB in DMF resulted in spontaneous coating of the PANB layers. TEM and high resolution-TEM images (HR-TEM) reveal that the as-obtained EEG-PANB possesses a similar morphology to the original EEG sheet, with no obvious PANB agglomerates detected on the EEG surface (Figure 2c).

The chemical structure of EEG-PANB and pristine EEG was investigated by Fouriertransform infrared (FT-IR) and Raman spectroscopy. In the FT-IR spectrum (Figure S3) of EEG-PANB, the peaks at 1581, 1496, 1311, 1168 and $830 \mathrm{~cm}^{-1}$ are consistent with the profiles of pure $\mathrm{PANB}$, corresponding to the vibration of the $-\mathrm{C}=\mathrm{N},-\mathrm{C}=\mathrm{C},-\mathrm{C}-\mathrm{N}$ and $-\mathrm{C}-\mathrm{H}$ inplane bending and out-of-plane deformation, respectively. ${ }^{23,24}$ In the Raman spectra of EEGPANB (Figure 2d), three representative peaks arising from PANB can be observed at 1163, 1224 and $1484 \mathrm{~cm}^{-1}$ in addition to the D, G and 2D band of EEG which are associated with the $\mathrm{C}-\mathrm{H}$ vibrations and the $\mathrm{C}-\mathrm{N}, \mathrm{C}=\mathrm{N}$ stretching of PANB chains. ${ }^{25}$ Notably, the G-band of EEG-PANB $\left(1568 \mathrm{~cm}^{-1}\right)$ is downshifted by $12 \mathrm{~cm}^{-1}$ compared with that of EEG $\left(1580 \mathrm{~cm}^{-1}\right)$ (inset of Figure 2d), suggesting strong electronic coupling between EEG and PANB (n-type doping of EEG). ${ }^{26} \mathrm{X}$-ray photoelectron spectroscopy (XPS) was used to probe the chemical compositions of EEG-PANB. The XPS survey spectra reveal the presence of C1s, O1s, and N1s (Figure 2e). Typically, EEG-PANB has an atomic content of $88.9,8.0$ and $3.1 \%$ for carbon, oxygen and nitrogen, respectively. The complex N1s spectra can be further deconvoluted into three signals with binding energies of $398.8,399.9$ and $402.0 \mathrm{eV}$ corresponding to $=\mathrm{N}-,-\mathrm{NH}-$ and $-\mathrm{N}^{+}-$, respectively (inset of Figure $2 \mathrm{e}$ ). ${ }^{27}$ The coating process of PANB is controllable by varying the concentration of PANB in DMF which is described in detail in the Supporting Information (Figure S5 and Table S1).

Electrical Property of EEG-PANS. The electrical conductivity of EEG-PANB before and after protonic acid doping was examined. Because the acid concentration plays an essential 
role for the degree of protonation of PANB, ${ }^{28,29}$ EEG-PANB was treated with $\mathrm{HCl}$ at different concentrations (e.g., $0.1 \mathrm{M}, 0.5 \mathrm{M}$ and $1.0 \mathrm{M}$ ) to generate EEG-emeraldine salt sheets (denoted as EEG-PANS-X, where $\mathrm{X}$ represents the $\mathrm{HCl}$ concentration). Figure $2 \mathrm{f}$ compares the resistance of the EEG, EEG-PANB and EEG-PANS-X films $\left(R_{\mathrm{f}}\right)$ obtained by the fourpoint probe method. The sample films (with an average thickness of $1 \mu \mathrm{m}$ ) on $\mathrm{SiO}_{2} / \mathrm{Si}$ substrates were prepared by a vacuum filtration and dry transfer method (see detailed procedure in the Supplementary Information). The EEG film exhibits an average $R_{\mathrm{f}}$ as low as $62.3 \Omega \mathrm{sq}^{-1}$. After functionalization with PANB, the $R_{\mathrm{f}}$ of EEG-PANB substantially increases to $21.0 \mathrm{k} \Omega \mathrm{sq}^{-1}$. This finding can be attributed to the PANB barrier layers resulting in perturbation of the charge transport of the EEG sheets. Remarkably, the $R_{\mathrm{f}}$ decreases dramatically when EEG-PANB is subjected to protonation with $\mathrm{HCl}$. Particularly, the average $R_{\mathrm{f}}$ of EEG-PANS-X decreases from $9.2 \mathrm{k} \Omega \mathrm{sq}^{-1}$ to $0.19 \mathrm{k} \Omega \mathrm{sq}^{-1}$ as the $\mathrm{HCl}$ concentration increases from $0.1 \mathrm{M}$ to $1.0 \mathrm{M}$. Although EEG-PANS-1 exhibits a higher $R_{\mathrm{f}}$ compared with the pristine EEG film, this value remains well below that of the chemically reduced GO film $\left(6.5 \mathrm{k} \Omega \mathrm{sq}^{-1}\right)$.

Assembly Procedure and Characterization of EEG-Si. Given that PANB provides abundant amine and imine groups to the EEG basal planes, the assembly of EEG-PANB with functional components is expected to produce EEG-based hybrid materials with defined nanostructures. For example, EEG-Si sandwich-like hybrids were synthesized by the coassembly of EEG-PANB nanosheets and commercial Si NPs (with an average particle size of $\leq 50 \mathrm{~nm}$ ) in DMF solution. Si NPs possess a high content of hydroxyl groups on the surface $\mathrm{SiO}_{2}$ layers ${ }^{30}$ which facilitates the coupling of Si NPs with EEG-PANB via electrostatic interactions and hydrogen bonding. The photograph illustrates the synthetic procedure leading to EEG-Si hybrids (Figure 3a). Interestingly, agglomerates spontaneously formed immediately when the Si NPs (Sample 2) were diluted in the EEG-PANB suspensions (Sample 1) suggesting that the self-assembly of the two species occurred. Under an optimal mass ratio, nearly all of EEG-PANB and Si NPs co-assembled, leaving a transparent solution (Sample 3). TEM and HR-TEM images (Figure 3b and 3c) verified the successful formation of EEG-Si, indicating that the Si NPs homogeneously adhered to the EEG surface. In a control experiment, Si NPs were mixed with a pristine EEG dispersion in the absence of PANB functionalization. However, only a dark-yellow suspension was obtained, as displayed in Sample 4. These comparative results highlight that the PANB functional layers act as the crucial driving force to couple the Si NPs to the EEG surface. 


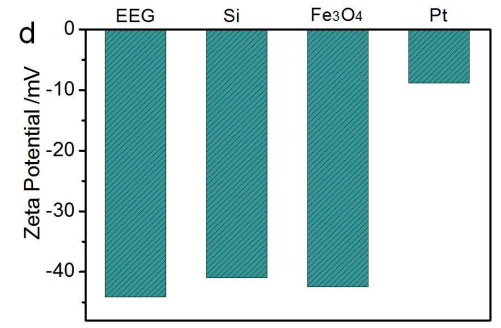

b
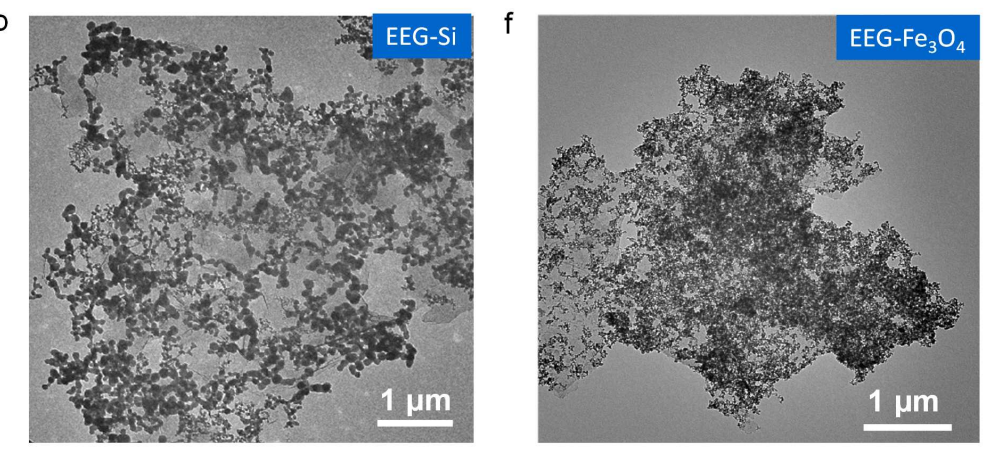

c
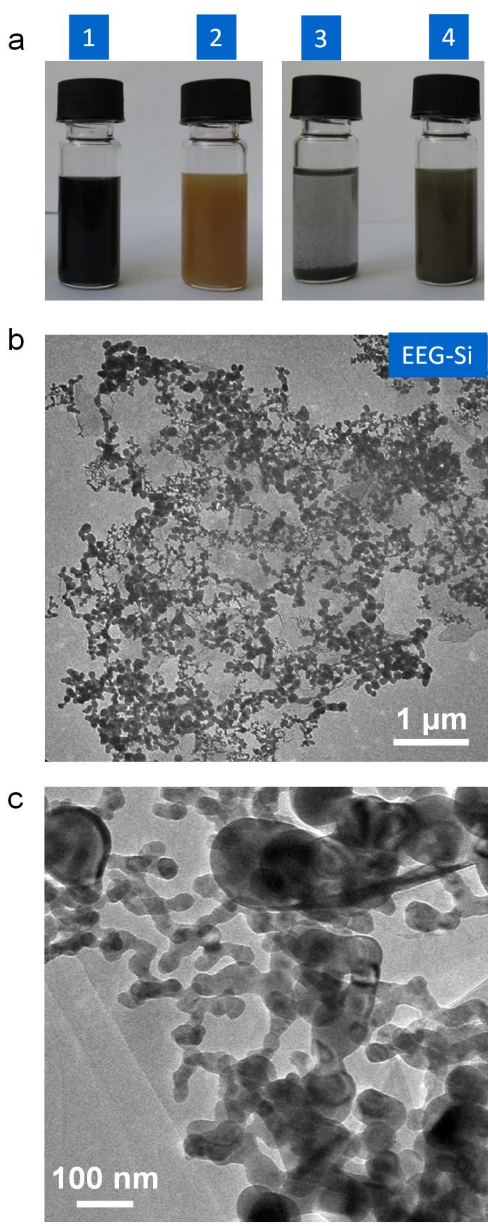

g

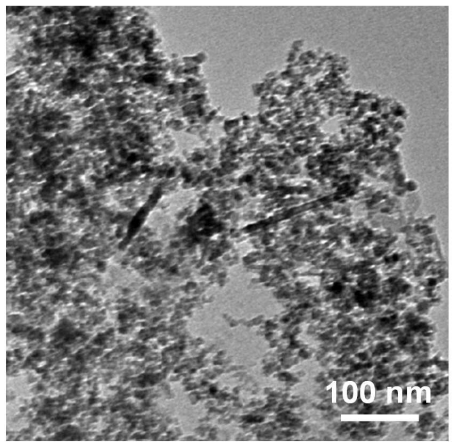

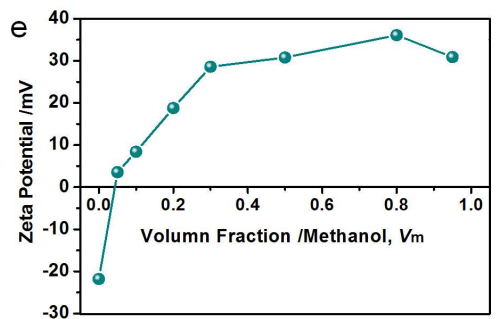

$\mathrm{h}$
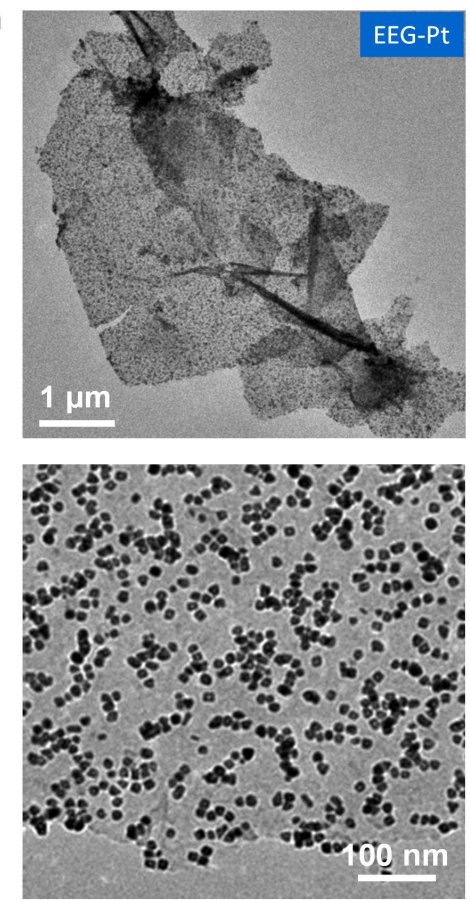

Figure 3. (a) Photographic illustration of the fabrication of EEG-Si hybrids (1. EEG-PANB in DMF; 2. Si NPs in DMF; 3. EEG-Si obtained after mixing samples 1 and 2; 4. Suspensions of pristine EEG sheets and Si NPs); (b) Typical TEM and (c) HR-TEM images of EEG-Si hybrids; (d) Zeta potential measurement of EEG and various colloidal nanoparticles (including $\mathrm{Si}, \mathrm{Fe}_{3} \mathrm{O}_{4}$, and $\mathrm{Pt}$ ); (e) Surface charge investigation of EEG-PANB in DMF solutions with various methanol volume fractions; (f-i) TEM and HR-TEM images of EEG-Fe $\mathrm{O}_{4}$ (f, g) and EEG-Pt hybrids (h, i).

Assembly Mechanism. To determine the mode of interactions between EEG-PANB and Si NPs, the surface charges of Si NPs and EEG before and after functionalization with PANB were monitored by zeta potential $(\zeta)$ measurements (Figure 3d). Typically, EEG nanosheets and Si NPs are negatively charged in DMF solutions $(\zeta=-21.8 \mathrm{mV}$ and $-41.1 \mathrm{mV}$, respectively). This result suggests that the formation of stable EEG/DMF dispersions can be partially attributed to the electrostatic repulsion which is similar to the colloidal behavior of GO in aqueous solution. ${ }^{31}$ To simulate the assembly process, we explored the surface charge 
of EEG-PANB in DMF solutions with various methanol volume fractions $\left(V_{\mathrm{m}}, 0-0.95\right)$. Methanol serves as a "soft" reagent to interact with EEG-PANB, having the same functionality as the hydroxyl groups on the Si NP surface. Figure 3e reveals that the surface charge of EEG-PANB has changed from negative $\left(\zeta=-21.8 \mathrm{mV}, V_{\mathrm{m}}=0\right)$ to positive $(\zeta=3.6$ $\left.\mathrm{mV}, V_{\mathrm{m}}=0.05\right)$ and increased linearly upon increasing $V_{\mathrm{m}}$ to $0.3(\zeta=28.6)$. Further addition of methanol results in a slight increase of the $\zeta$ value. We surmise that as more hydroxyl groups are present in the dispersed phase (upon addition of Si NPs or methanol), more imine nitrogen atoms in the PANB chains are protonated, leading to an increase of positive charges on the PANB chains and the EEG surface. Subsequently, the mutual assembly can be readily triggered when the positively charged EEG-PANB interacts with the negatively charged $\mathrm{Si}$ NPs.

This co-assembly protocol could be further adapted to construct other 2D EEG-CNP hybrids by employing different functional CNPs such as $\mathrm{Fe}_{3} \mathrm{O}_{4}$ and polyvinylpyrrolidone (PVP)-capped Pt NPs. The synthetic procedures toward $\mathrm{Fe}_{3} \mathrm{O}_{4}$ and PVP-capped Pt NPs are described in the Supplementary Information. The resultant $\mathrm{Fe}_{3} \mathrm{O}_{4}$ and $\mathrm{Pt}$ NPs have average particle sizes of $\sim 10$ and $\sim 12 \mathrm{~nm}$, respectively (Figure S8). Further, TEM images of EEGCNPs (Figure 3f-i) demonstrate that in each case the CNPs are uniformly decorated on the EEG surface. Similar to Si NPs, the $\mathrm{Fe}_{3} \mathrm{O}_{4}$ and Pt NPs are negatively charged because of ionization of the surface functionalities (Figure 3d). These functional groups protonate the imine side chains of PANB and promote assembly with the EEG-PANB sheets. In the case of Pt NPs stabilized by neutral capping ligands (PVP) with a low charge density $(\zeta=-9.0 \mathrm{mV})$, we assume that the interaction is primarily driven by the hydrogen bonding between PANB and PVP rather than by electrostatic interactions. ${ }^{32,33}$ 

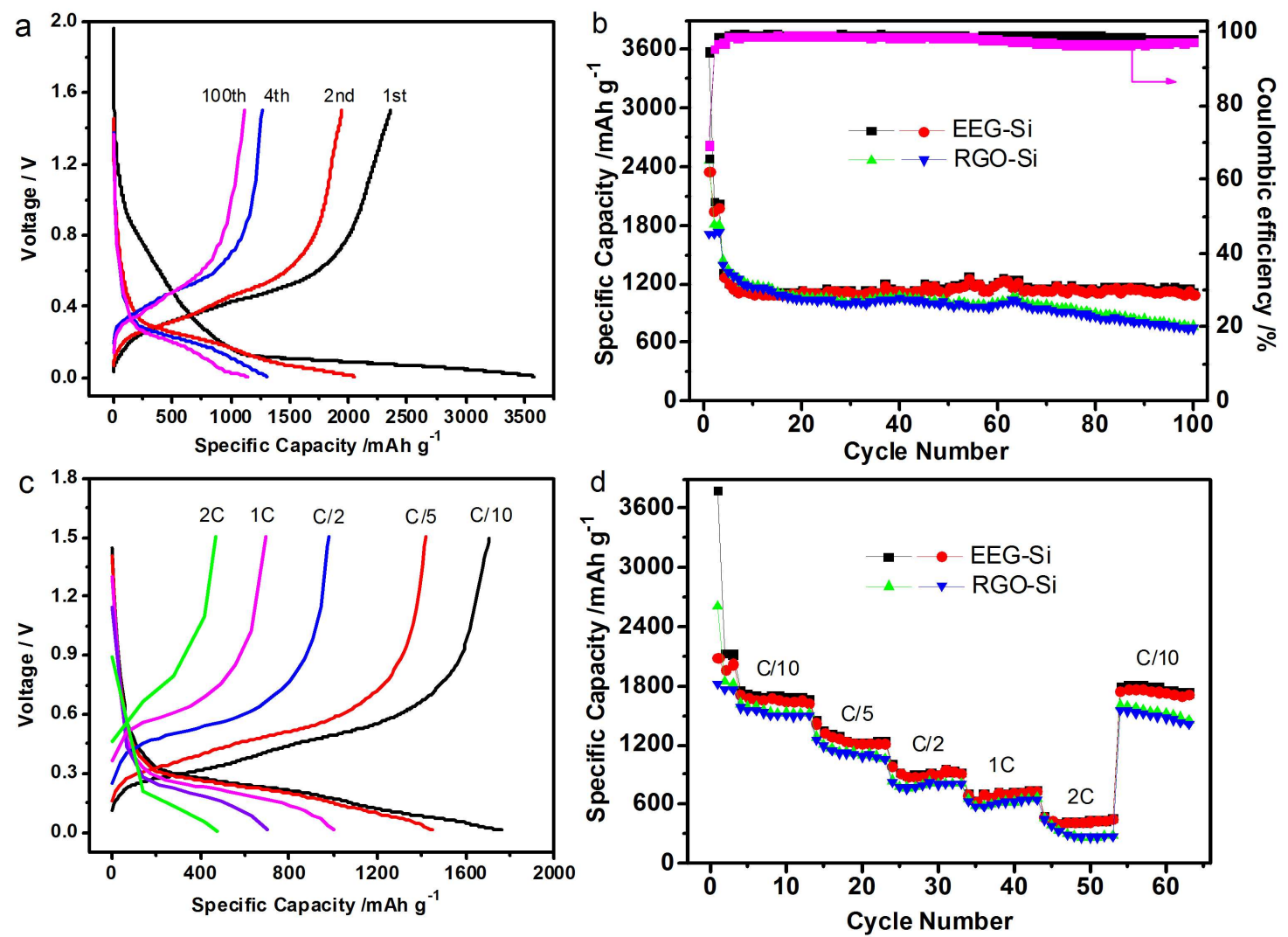

Figure 4. (a) Galvanostatic cycling profiles of EEG-Si for the 1st, 2nd, 4th and 100th cycle; (b) Cycling performance and Coulombic efficiency of EEG-Si and RGO-Si hybrids; (c) Galvanostatic cycling profiles of EEG-Si at various current densities; (d) Rate capability of EEG-Si and RGO-Si electrodes. The current densities of the three initial cycles for all of the electrodes are 105,210 , and $210 \mathrm{~mA} \mathrm{~g}^{-1}$.

EEG-Si as Anode Material for Lithium Storage. Graphene-based hybrids hold great promise as electrode materials for energy storage applications such as lithium-ion batteries (LiBs). ${ }^{34}$ Recently, the design and fabrication of graphene/silicon anode materials have attracted immense attention because of the combination of high-capacity $\mathrm{Si}$ active materials (4200 $\mathrm{mAh} \mathrm{g}^{-1}$ ) and the unique graphene matrix. ${ }^{35,36}$ In addition to improving electrical conductivity, graphene layers afford an elastic buffering space to accommodate the volume change of Si during the lithiation/delithiation process. ${ }^{30,37}$ However, the RGO/Si NPs hybrids prepared through either the mixing/filtration or assembly approach suffer from low electrochemical performance, primarily because of the non-uniform dispersion of Si NPs and the limited contact with graphene. ${ }^{38,39}$ In this study, monodisperse Si NPs conformally bonded with the EEG sheets, forming an Si NP structural support and promising efficient transport pathways. To determine the effect of EEG on electrochemical performance, 2D RGO-derived Si hybrids (RGO-Si) were prepared for comparison. RGO-Si was prepared by the same 
synthetic approach using RGO as the building block (see detailed procedure in the Supporting Information).

The electrochemical properties of EEG-Si hybrids were first examined by cyclic voltammetry (CV) (Figure S9). Similar to the pristine Si powders, the peaks at 0.01 and 0.16 $\mathrm{V}$ in the cathodic process correspond to the conversion of crystalline Si to the LixSi phase, while the two peaks at 0.35 and $0.52 \mathrm{~V}$ in the anodic process correspond to the delithiation of amorphous LixSi to $\mathrm{Si}^{40}$ The electrochemical cycling performance of the EEG-Si and RGOSi electrode was evaluated using discharge/charge galvanostatic cycling over the potential of 0.01-1.5 V versus $\mathrm{Li} / \mathrm{Li}^{+}$. The capacity values reported in this study are based on the total weight of active material. As shown in Figures $4 \mathrm{a}$ and $\mathrm{b}$, the EEG-Si electrode delivers a reversible capacity of 2357 and $2048 \mathrm{mAh} \mathrm{g}^{-1}$ in the first and second cycles at a current density of 105 and $210 \mathrm{~mA} \mathrm{~g}^{-1}$ respectively which are higher than those of the RGO-Si electrode (1709 and $1718 \mathrm{mAh} \mathrm{g}^{-1}$, respectively). Based on the silicon mass ratio (78\%, Figure S10) in EEG-Si hybrids, the capacity with respect to silicon is calculated as 3022 and $2660 \mathrm{mAh} \mathrm{g}^{-1}$. From the 4th cycle, the discharge/charge test was performed at a high current rate of $\sim 1 \mathrm{~A} \mathrm{~g}^{-1}$. Remarkably, the EEG-Si electrode exhibits a substantially better cycling performance than the RGO-Si electrode. After 100 cycles, the reversible capacity of EEG-Si remains as high as $1310 \mathrm{mAh} \mathrm{g}^{-1}$ which corresponds to $86 \%$ capacity retention, while that of RGO-Si is only $748 \mathrm{mAh} \mathrm{g}^{-1}$ (capacity retention of 53\%). Accordingly, a higher Coulombic efficiency of EEG-Si (above 97\% after the initial three cycles) than that of RGO-Si is achieved.

The EEG-Si sample possesses a superior rate capability to the RGO-Si electrode. As the current density increases from 0.42 to $4.2 \mathrm{~A} \mathrm{~g}^{-1}$, the EEG-Si electrode displays reversible and stable capacities of 1710 and $720 \mathrm{mAh} \mathrm{g}^{-1}$, respectively. At a high current density of $8.4 \mathrm{~A} \mathrm{~g}^{-1}$, the EEG-Si electrode still delivers a favorable capacity of $460 \mathrm{mAh} \mathrm{g}{ }^{-1}$, while RGO-Si exhibits a capacity of $275 \mathrm{mAh} \mathrm{g}^{-1}$. To verify the improved electrochemical performance of EEG-Si compared with RGO-Si, the structure and morphology of both of the electrodes after the 80th cycle were examined by scanning electron microscopy. Because EEG-Si and RGO-Si were produced by the same assembly procedure, the electrode materials show a similar morphology with a uniform solid-electrolyte interphase (SEI) formed on the hybrid surface (Figure S11a, b). SEM images indicate that both electrode films exhibit cracks due to the extreme volume change of the $\mathrm{Si}$ active material (Figure $\mathrm{S} 11 \mathrm{c}, \mathrm{d}$ ). Notably, the RGO-Si electrode film underwent severe cracking and disassembly of the active material compared 
with that of the EEG-Si electrode, leading to loss of electrical contact and eventual fading of capacity. This comparative result indicates that the EEG nanosheets can better withstand the stress buildup within the $\mathrm{Si}$ anode compared with RGO, primarily because of the excellent mechanical properties of EEG. ${ }^{16}$ This stabilizing effect of the EEG substrates was further confirmed by electrochemical impedance spectroscopy (EIS) measurements. Nyquist plots (Figure S12) showed that the diameter of the semicircle for the EEG-Si electrode in the highmedium frequency region is much smaller than that of RGO-Si, suggesting reduced contact and charge-transfer impedances of EEG-Si. The kinetic parameters of both electrodes were simulated via a Randles equivalent circuit model (Figure S13 and Table S2). Remarkably, the EEG-Si electrode exhibits significant lower SEI film resistance $\left(R_{\mathrm{f}}=16.7 \Omega\right)$ and chargetransfer resistance $\left(R_{\mathrm{ct}}=32.2 \Omega\right)$ than those of RGO-Si (39.2 and $101.0 \Omega$ respectively), which highlights an improved electrical conductivity of EEG-Si electrode.

\section{- CONCLUSION}

In summary, a synthetic concept utilizing electrochemically exfoliated graphene (EEG) for the assembly of 2D nanohybrids was established in an attempt to promote the processability and potential applications of EEG. Polyaniline as emeraldine base (PANB) was used as a powerful dopant for the functionalization of EEG and the controlled assembly of functional nanoparticles. The capability to manipulate EEG hybrid structure is advantageous in the general context of graphene materials and their assemblies, including the versatility for integrating functional components, the use of inexpensive ingredients and the simple processing. Therefore, the novel bottom-up approach described here provides access to the development of a wide range of interesting, scalable nanostructured EEG materials with desired functionalities for diverse applications.

\section{- ASSOCIATED CONTENT}

\section{Supporting Information}

This material is available free of charge via the Internet at http://pubs.acs.org: experimental details, chemical structure of PANB and PANS, SEM and TEM images of EEG, TEM images of EEG-PANB, $\mathrm{Fe}_{3} \mathrm{O}_{4}$ and Pt NPs, FT-IR of EEG and EEG-PANB, XRD of EEG and EEG-Si, XPS spectra of EEG, EEG-PANB and EEG-Si, TGA of EEG-CNP hybrids; comparison of elemental analysis of EEG-PANB samples; CV curves of EEG-Si anode; and Nyquist plots of EEG-Si and RGO-Si anodes. 


\section{AUTHOR INFORMATION}

\section{Corresponding Author}

xinliang.feng@tu-dresden.de

muellen@mpip-mainz.mpg.de

Notes

The authors declare no competing financial interests.

\section{- ACKNOWLEDGMENTS}

This study was financially supported by the ERC grants for NANOGRAPH and 2DMATER, the DFG Priority Program SPP 1459, the EC under the Graphene Flagship (grant number CNECT-ICT-604391), and the EU GENIUS and UPGRADE Projects. The authors thank Dr. Rafael Muñoz-Espí for the help with zeta potential measurement and Dr. Xiaodong Zhuang for XPS measurement.

\section{- REFERENCES}

(1) Geim, A. K.; Novoselov, K. S. Nat. Mater. 2007, 6, 183.

(2) Huang, X.; Tan, C.; Yin, Z.; Zhang, H. Adv. Mater. 2014, 26, 2185.

(3) Wei, W.; Liang, H.; Parvez, K.; Zhuang, X.; Feng, X.; Müllen, K. Angew. Chem. Int. Ed. 2014, 53, 1570.

(4) Lee, S. H.; Lee, D. H.; Lee, W. J.; Kim, S. O. Adv. Funct. Mater. 2011, 21, 1338.

(5) Yang, S.; Feng, X.; Ivanovici, S.; Müllen, K. Angew. Chem. Int. Ed. 2010, 49, 8408.

(6) Yang, S.; Feng, X.; Wang, L.; Tang, K.; Maier, J.; Müllen, K. Angew. Chem. Int. Ed. 2010, 49, 4795 .

(7) Wei, W.; Yang, S.; Zhou, H.; Lieberwirth, I.; Feng, X.; Müllen, K. Adv. Mater. 2013, 25, 2909.

(8) Dreyer, D. R.; Park, S.; Bielawski, C. W.; Ruoff, R. S. Chem. Soc. Rev. 2010, 39, 228.

(9) Georgakilas, V.; Otyepka, M.; Bourlinos, A. B.; Chandra, V.; Kim, N.; Kemp, K. C.;

Hobza, P.; Zboril, R.; Kim, K. S. Chem. Rev. 2012, 112, 6156.

(10) He, H.; Klinowski, J.; Forster, M.; Lerf, A. Chem. Phys. Lett. 1998, $287,53$.

(11) Stankovich, S.; Dikin, D. A.; Piner, R. D.; Kohlhaas, K. A.; Kleinhammes, A.; Jia, Y.;

Wu, Y.; Nguyen, S. T.; Ruoff, R. S. Carbon 2007, 45, 1558.

(12) Zhong, Y. L.; Swager, T. M. J. Am. Chem. Soc. 2012, 134, 17896.

(13) Wang, J.; Manga, K. K.; Bao, Q.; Loh, K. P. J. Am. Chem. Soc. 2011, 133, 8888.

(14) Liu, N.; Luo, F.; Wu, H.; Liu, Y.; Zhang, C.; Chen, J. Adv. Funct. Mater. 2008, 18, 1518. 
(15) Parvez, K.; Li, R.; Puniredd, S. R.; Hernandez, Y.; Hinkel, F.; Wang, S.; Feng, X.; Müllen, K. ACS Nano 2013, 7, 3598.

(16) Parvez, K.; Wu, Z.-S.; Li, R.; Liu, X.; Graf, R.; Feng, X.; Müllen, K. J. Am. Chem. Soc. 2014, 136, 6083.

(17) Wang, G.; Wang, B.; Park, J.; Wang, Y.; Sun, B.; Yao, J. Carbon 2009, 47, 3242.

(18) Stankovich, S.; Dikin, D. A.; Dommett, G. H. B.; Kohlhaas, K. M.; Zimney, E. J.; Stach, E. A.; Piner, R. D.; Nguyen, S. T.; Ruoff, R. S. Nature 2006, 442, 282.

(19) Stankovich, S.; Piner, R. D.; Chen, X.; Wu, N.; Nguyen, S. T.; Ruoff, R. S. J. Mater. Chem. 2006, 16, 155.

(20) MacDiarmid, A. G. Angew. Chem. Int. Ed. 2001, 40, 2581.

(21) Ćirić-Marjanović, G. Synth. Met. 2013, 170, 31.

(22) Pron, A.; Rannou, P. Prog. Polym. Sci. 2002, 27, 135.

(23) Li, Y.; Fang, Y.; Liu, H.; Wu, X.; Lu, Y. Nanoscale 2012, 4, 2867.

(24) Kim, D.; Choi, J.; Kim, J.-Y.; Han, Y.-K.; Sohn, D. Macromolecules 2002, 35, 5314.

(25) Wang, D.-W.; Li, F.; Zhao, J.; Ren, W.; Chen, Z.-G.; Tan, J.; Wu, Z.-S.; Gentle, I.; Lu, G. Q.; Cheng, H.-M. ACS Nano 2009, 3, 1745.

(26) Su, Q.; Pang, S.; Alijani, V.; Li, C.; Feng, X.; Müllen, K. Adv. Mater. 2009, 21, 3191.

(27) Tan, K. L.; Tan, B. T. G.; Kang, E. T.; Neoh, K. G. Phys. Rev. B 1989, 39, 8070.

(28) MacDiarmid, A.; Chiang, J.; Richter, A.; Epstein, A. Synth. Met. 1987, 18, 285.

(29) Chiang, J.-C.; MacDiarmid, A. G. Synth. Met. 1986, 13, 193.

(30) Wu, H.; Yu, G.; Pan, L.; Liu, N.; McDowell, M. T.; Bao, Z.; Cui, Y. Nat. Commun. 2013, 4 .

(31) Li, D.; Müller, M. B.; Gilje, S.; Kaner, R. B.; Wallace G. G. Nat. Nanotechnol. 2008, 3, 101.

(32) Cruz-Silva, R.; Arizmendi, L.; Del-Angel, M.; Romero-Garcia, J. Langmuir 2006, 23, 8. (33) Lei, Z.; Zhao, M.; Dang, L.; An, L.; Lu, M.; Lo, A.-Y.; Yu, N.; Liu, S.-B. J. Mater.

Chem. 2009, 19, 5985.

(34) Huang, X.; Qi, X.; Boey, F.; Zhang, H. Chem. Soc. Rev. 2012, 41, 666.

(35) Ji, J.; Ji, H.; Zhang, L. L.; Zhao, X.; Bai, X.; Fan, X.; Zhang, F.; Ruoff, R. S. Adv. Mater. 2013, 25, 4673 .

(36) Evanoff, K.; Magasinski, A.; Yang, J.; Yushin, G. Adv. Energy Mater. 2011, 1, 495.

(37) Zhang, F.; Yang, X.; Xie, Y.; Yi, N.; Huang, Y.; Chen, Y. Carbon 2015, 82, 161.

(38) Zhou, X.; Yin, Y.-X.; Wan, L.-J.; Guo, Y.-G. Chem. Commun. 2012, 48, 2198. 
(39) Lee, J. K.; Smith, K. B.; Hayner, C. M.; Kung, H. H. Chem. Commun. 2010, 46, 2025.

(40) Magasinski, A.; Dixon, P.; Hertzberg, B.; Kvit, A.; Ayala, J.; Yushin, G. Nat. Mater. 2010, 9, 353. 


\section{TOC graphic}

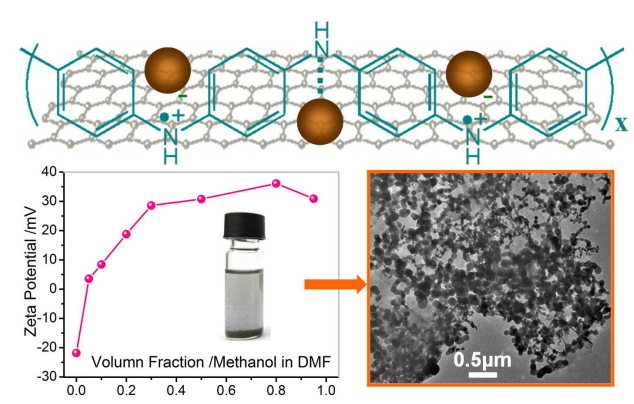

15
16
17

17

18

19

20

21

22

23

24

25

26

27

28

29

30

31

32

33

34

35

36

37

38

39

40

41

42

43

44

45

46

47

48

49

50

51

52

53

54

55

56

57

58

59 